\title{
Reduction of Bitterness and Enhancing Palatability of Cetirizine Oral Liquid Dosage Forms by Cyclodextrins
}

\author{
Ali $\mathrm{M}^{1}$, EL-Massik MA ${ }^{1}$, Abdallah $\mathrm{OY}^{2}$ and Abdelkader $\mathrm{H}^{{ }^{3}}$ \\ ${ }^{1}$ Department of Pharmaceutics, Faculty of Pharmacy, Pharos University, Alexandria, Egypt \\ ${ }^{2}$ Department of Pharmaceutics, Faculty of Pharmacy, Alexandria University, Alexandria, Egypt \\ ${ }^{3}$ Department of Pharmaceutics, Faculty of Pharmacy, Minia University, Minia, Egypt
}

${ }^{*}$ Corresponding author: Abdelkader H, PhD, Department of Pharmaceutics, Faculty of Pharmacy, Minia University, Minia, Egypt, Fax: 0020862369075, Tel: 00201275119494, E-mail: hamdy2002m@yahoo.com, h.abdelkader@mu.edu.eg

Citation: Ali M, EL-Massik MA, Abdallah OY, Abdelkader H (2014) Reduction of Bitterness and Enhancing Palatability of Cetirizine Oral Liquid Dosage Forms by Cyclodextrins. J Pharm Drug Devel 2(1): 102. doi: 10.15744/2348-9782.1.302

Received Date: November 26, 2013 Accepted Date: March 6, 2014 Published Date: March 11, 2014

\begin{abstract}
The aim of this manuscript is to study cyclodextrins (CDs) as a potential excipient to suppress bitterness and enhance palatability of pediatric liquid preparations for Cetirizine, an extremely bitter drug. Natural $\alpha, \beta$ and $\gamma$ CDs; and $\beta$ CD derivatives such as hydroxyl propyl (HP), randomly methylated (RM) and sulfobutyl ether (SBE) $\beta$-CDs were screened in different molar ratios of 1:1, 1:2 and 1:3 for their inhibition of the extremely bitter taste of Cetirizine using the human gustatory sensation test. Cavity size and chemical substitution are dominantly two factors that affect Cetirizine inclusion into natural CDs and $\beta$ CD derivatives respectively. Syrup and drops formulations were prepared based on the selected $\alpha-, \gamma$-and RM $\beta$-CDs. The prepared oral liquids were evaluated for palatability, and antimicrobial stability under stress conditions of $40{ }^{\circ} \mathrm{C}$ and $75 \%$ relative humidity (RH) for three months. Potasium sorbate was found to be an effective preservative for CDs-based oral liquids. RM $\beta$-CD-based oral liquids demonstrated dramatic reduction of the bitterness of drops and can be administered without prior dilution as recommended by the manufacturer.

Keywords: Cetirizine; Cyclodextrins; Bitterness; Palatability; Stability
\end{abstract}

\section{Introduction}

Cetirizine (Figure 1) is an orally active metabolite of hydroxyzine, a piperazine H1-receptor antagonist. However, it differs from the parent compound by having greater affinity for the H1-receptor. Cetirizine has a lower incidence of sedation compared with older antihistamines. Drowsiness may, nevertheless, be dose-related. Cetirizine is effective in the treatment of chronic idiopathic urticaria, perennial and seasonal allergic rhinitis, allergic asthma, physical urticaria (triggered by physical stimuli), and for symptomatic relief of atopic dermatitis [1]. Cetirizine occurs as a racemic mixture with levocetirizine being the active enantiomer. Cetirizine can be prescribed for pediatric and elderly patients. An expansion of labeling for use in infants as young as 6 months of age in 2002 was FDA-approved [2]. However, Cetirizine has an extremely bitter taste and this might pose patient incompliance due to poor palatability among pediatric patients. This problem is noticeable with the marketed drops dosage form where the concentration of Cetrizine $(10 \mathrm{mg} / \mathrm{ml})$ is 10 times higher than the syrup dosage form $(1 \mathrm{mg} / \mathrm{ml})$.

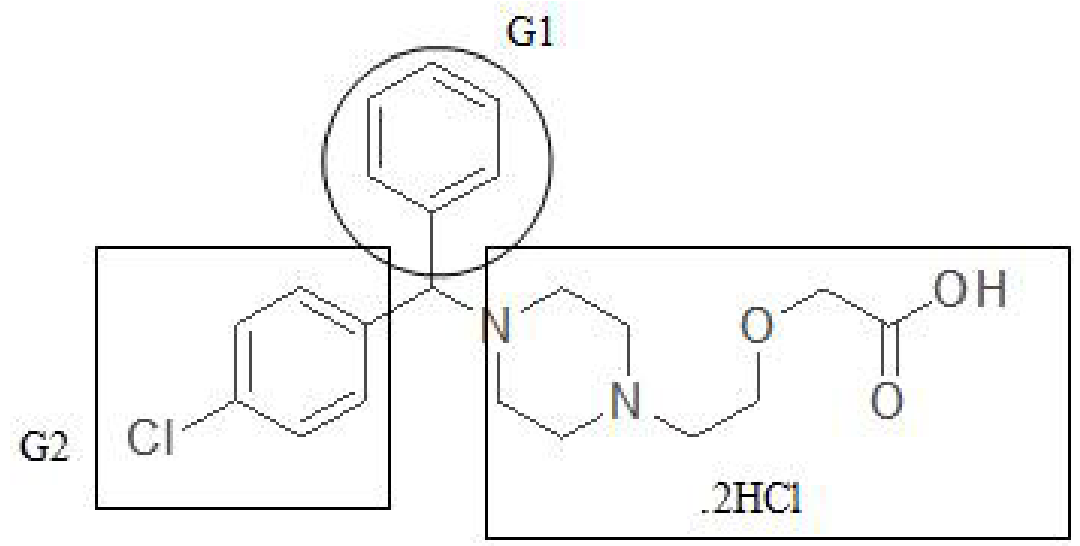

Figure 1: Chemical structure of Cetirizine showing the different groups (G1, G2 \& G3). 
Cetirizine is administered orally and the bioavailability of the tablets and oral syrup is comparable. It has a rapid onset (i.e., $\mathrm{t}_{\max }$ 1 hour in adults) and a long duration of action. The overall bioavailability of Cetirizine is not altered by the presence of food, although the rate of absorption can be slightly reduced [1].

Many efforts have been reported in an attempt to decrease the bitter taste of Cetirizine [3-5]. Cetirizine chewable tablets with inhibited bitterness using the lipophilic Gelucire 33/01 in presence of soybean lecithin were reported to markedly reduce the bitterness of the drug without affecting the drug release [4].

European Patent Application No. EP 0409254 [6] disclosed oral taste masked solid preparations of Cetirizine, masked via film layer coating of core tablets. The film layer contained a mouth insoluble polymers as ethyl cellulose. The effective masking time was described as from 20-60 seconds. However, such time period may not be enough to provide complete taste masking for some patients such as those with swallowing difficulties, children and elderly. Also, they cannot avoid the use of organic solvents (e.g., methylene chloride and acetone) which are associated with toxicological and safety related issues.

European patent No. EP. 548,356 [7] presented multiparticulate tablets having a disintegration rate in the oral cavity or on the tongue of less than 60 seconds which contain the active ingredient in the form of coated microcrystals, particularly microgranules for masking the taste of Cetirizine.

For oral liquids intended for pediatric patients administration, palatability of the dosage forms is essentially important factor determining patient acceptability and compliance. Pediatric patients are not easily convinced and do not understand the need to take medicines, and are likely to refuse to take them, if they have an unpleasant taste or smell. In particular, a bitter taste may give rise to a dramatic decrease of compliance [8]. Therefore, it has been proposed that the selection and/or administration of medicines for pediatric patients should take bitterness and palatability into consideration [8].

Syrups are concentrated aqueous solutions of sucrose $(60 \%-80 \% \mathrm{w} / \mathrm{v})$, other sugars or sweetening agents that are widely used as vehicles for their flavouring and sweetening properties the vehicle of choice especially for children and elderly patients. However, syrup is not stable for long storage, and taste masking. Generally, syrup and drops formulations are accompanied by many problems concerning their physical nature, where formulation in aqueous solution form increases the possibilities of degradation and interaction of the formulation components. In aqueous formulations containing drug-CD complex, the complex is present in the equilibrium state, continually dissociating and associating leading by time to possible precipitation of the drug, if used in an amount exceeding its intrinsic solubility. In addition, competition problems from other formulation ingredients, such as preservatives (that may displace the drug from the $\mathrm{CD}$ complex) may lead to serious instability problems as precipitation or even degradation of the drug together with a decrease or loss of the antimicrobial activity of the preservative. The structure of Cetirizine can simply be divided into three distinct groups, the phenyl group (G1), the chlorophenyl group (G2) and the piperazinyl ethoxy acetic acid, dihydrochloride group (G3) as shown in Figure 1. The first two groups (G1 and G2) are hydrophobic in nature and may contribute to inclusion complexation but the third group (G3) is hydrophilic. This group then may not favor the interior hydrophobic cavity of the $\mathrm{CD}$ but will probably favor the exterior hydrophilic medium.

The aim of this manuscript is to prepare and characterize oral liquid formulations (drops and syrups) in an attempt to improve bitterness scores of Cetirizine and maintain their palatability over extended period of time using cyclodextrins. Specific objectives will look at the effect of some selected cyclodextrins on the bitterness scores for Cetirizine oral liquid formulations (syrups and drops) adopting the human gustatory test, and stability of the prepared formulations in terms of changes in the taste masking potential and $\mathrm{pH}$ of the prepared liquid dosage forms under stress conditions.

\section{Materials and Methods}

\section{Materials}

Cetirizine dihydrochloride was supplied by Dr Reddy, India. $\alpha$-cyclodextrin (CD), and $\gamma$-CD were supplied by Wacher Chemie, Germany. Hydroxy propyl (HP) $\beta$-CD and sulfobutyl ether (SBE) $\beta$-CD and randomly methylated (RM) $\beta$ - CD, were purchased from Hebi Smart Chemicals, China. Quinine hydrochloride (BP grade) was purchased from Sigma-Aldrich, USA. Propylene glycol was purchased from SK-Networks, Korea and potassium sorbate was purchased from El-Gomhoria, Egypt. Innovator syrup and drops: Zyrtec ${ }^{\bullet} \operatorname{syrup}\left(1 \mathrm{mg} / \mathrm{ml}\right.$ of Cetirizine) and Zyrtec ${ }^{\circledR}$ drops (10 mg/ml of Cetirizine) (UCB, Egypt).

\section{Preliminary screening of cyclodextrins}

Test solutions of Cetirizine solution $(1 \mathrm{mg} / \mathrm{ml})$ alone and in presence of the different CDs $(\alpha-C D, \beta-C D, \gamma-C D, R M \beta-C D, H P$ $\beta-C D$ and SBE $\beta-C D$ ) in different Cetirizine: CD molar ratios of 1:1, 1:2 and 1:3 were prepared. These test solutions were studied for palatability and reduction of bitterness of Cetirizine, compared with standard quinine solutions as will be mentioned below under Human gustatory sensation test.

\section{Preparation of Cetirizine syrup $(\mathrm{F} 1 \rightarrow \mathrm{F} 4)$ and drops (F5 and F6) formulations}

Three syrup formulations, each containing $1 \mathrm{mg} / \mathrm{ml}$ Cetirizine were prepared in 1:3 molar ratios of Cetirizine to the natural CDs ( $\alpha$ - and $\gamma$-) or the RM $\beta$-CD derivative (Table 1 ). 
One formulation of Cetirizine drops containing $10 \mathrm{mg} / \mathrm{ml}$ Cetirizine and using the $\mathrm{RM} \beta$-CD derivative in 1:3 molar ratio (Table 1) was also prepared. Cetirizine was dissolved in distilled (dist) water. The selected CD ( $\alpha, \gamma$ or RM $\beta$-CD) was dissolved separately in dist water and poured into the Cetirizine solution under continuous stirring for 5 minutes. Table 1 summarizes the specified amounts of the drug and all other excipients. Glycerol was then added into the Cetirizine solution. Potassium sorbate was dissolved in Propylene glycol and added into the drug - CD mixtures. Sodium Hydroxide was added to adjust the $\mathrm{pH}$ from 4-5, then the volume was completed with water and the final solution was filtered. For comparison, a control formula without CD was prepared for syrup (F1) and drops (F5). The $\mathrm{pH}$ of the prepared formulations was measured using a $\mathrm{pH}$ meter (ORION, 420A, USA). All formulations were kept in a dark cool place till further use.

\begin{tabular}{|c|c|c|c|c|}
\hline \multirow{2}{*}{$\begin{array}{l}\text { Dosage form and Formula } \\
\text { code }\end{array}$} & \multirow[t]{2}{*}{ Selected CD } & \multicolumn{3}{|c|}{ Ingredients ${ }^{*}(\mathrm{mg})$} \\
\hline & & Cyclodextrin & Glycerol & Water \\
\hline \multicolumn{5}{|l|}{ Syrup } \\
\hline $\mathrm{F} 1$ & ----- & ----- & 1000 & To $5 \mathrm{ml}$ \\
\hline $\mathrm{F} 2$ & $\alpha-C D$ & 31.6 & 1000 & To $5 \mathrm{ml}$ \\
\hline F3 & $\gamma-\mathrm{CD}$ & 42.12 & 1000 & To $5 \mathrm{ml}$ \\
\hline $\mathrm{F} 4$ & $\mathrm{RM} \beta-\mathrm{CD}$ & 39.25 & 1000 & To $5 \mathrm{ml}$ \\
\hline \multicolumn{5}{|l|}{ Drops } \\
\hline F5 & ---- & ---- & 250 & To $1 \mathrm{ml}$ \\
\hline F6 & $\mathrm{RM} \beta-\mathrm{CD}$ & 74.5 & 250 & To $1 \mathrm{ml}$ \\
\hline
\end{tabular}

* All formulations contained potassium sorbate $1.346 \mathrm{mg} / \mathrm{ml}$ and propylene glycol $20 \mathrm{mg} / \mathrm{ml}$.

Table 1: Composition of Cetirizine syrups $(5 \mathrm{mg} / 5 \mathrm{ml})$ and drops $(10 \mathrm{mg} / \mathrm{ml})$ formulated with different types of cyclodextrins, in drug: CD molar ratio of 1:3.

\section{Determination of cetirizine in liquid dosage form}

A spectrophotometric method depending on the concept of the second derivative ratio of the absorbance spectra was adopted [9]. This is to eliminate any interference between Cetirizine and the excipients used that was observed with normal UV-spectrum absorption (zero derivatives).

\section{Preparation of test solutions}

Measured volumes of each syrup formulation $(10 \mathrm{ml})$ or drops formulation $(1 \mathrm{ml})$ were transferred into 100 -ml volumetric flasks and the volume made up to $100 \mathrm{ml}$ with dist water; stirred for $15 \mathrm{~min}$ and then filtered using $0.22 \mu \mathrm{m}$ filters. Two ml of each were then transferred into $50-\mathrm{ml}$ volumetric flasks and the volume was made up to $50 \mathrm{ml}$ with dist water to prepare test samples for syrup and drops (test ${ }_{\text {syrup }}$ and test drops ), respectively.

\section{Preparation of cetirizine standard solution}

Cetirizine powder $(50 \mathrm{mg}$ ) was transferred into a 100-ml volumetric flask, and the volume was made up to $100 \mathrm{ml}$ with dist water, then $2 \mathrm{ml}$ of the solution were further diluted to $50 \mathrm{ml}$ with dist water (stdcet.).

\section{Preparation of potassium sorbate (ps) standard solution}

Potassium sorbate $(134.6 \mathrm{mg})$ was dissolved in dist water and the volume was made up to $100 \mathrm{ml}$ in a 100 -ml volumetric flask. Aliquots of $10 \mathrm{ml}\left(\mathrm{std}_{\text {syrup }}\right)$ and $1 \mathrm{ml}\left(\mathrm{std}_{\text {drops }}\right)$ of this solution were diluted to $100 \mathrm{ml}$ with water. Two ml of each were further diluted to $50 \mathrm{ml}$ with water to prepare potassium sorbate (PS) standards for the drops and syrup (PS std drops ) and (PS std syrup $_{\text {) }}$ respectively.

\section{Sample analysis and measurements}

The UV spectra of all test and standard solutions were recorded using a UV-VIS Spectrophotometer (Shimadzu UV1601 PC, Japan). The absorption spectrum of each of test ${ }_{\text {drops }}$ and std $_{\text {cet }}$ were divided with the absorption spectrum of PS std drops in case of drops and test $_{\text {syrup }}$, std cet $_{\text {with PS std }}$ in in case of syrup. Then, the second derivative absorption spectra of test and standard ratios were recorded with delta lambda $=8$ and scaling factor $=100$. The drug content was determined using the peak to valley method of the second derivative ratio at $\lambda=232.6 \mathrm{~nm}$ and $242 \mathrm{~nm}$ respectively.

\section{Human gustatory sensation test}

This test was performed employing nine healthy and well-educated volunteers to screen the taste masking potential of the prepared syrup and drops formulations (test samples) for bitter taste intensity of Cetirizine, and compared with the standard (a well-known standard bitter drug) quinine hydrochloride solutions of $0.001,0.01,0.03,0.1,0.3$ and $1.0 \mathrm{mM}$ with well-defined bitterness scores of $0,1,2,3,4$ and 5 respectively [3]. Before testing, the volunteers were asked to drop $1 \mathrm{ml}$ of the standard quinine solutions onto the center of the tongue and kept in the mouth for 10 seconds and were told their bitterness scores. The mouth was then thoroughly rinsed with water. One $\mathrm{ml}$ of each test sample was dropped on the center of the tongue and kept in the mouth for 10 seconds. Then, 
the volunteers were asked to give a bitterness intensity scores for the test samples. After tasting each sample, the volunteers gargled well and waited for about 15 minutes before the next sample.

\section{Antimicrobial effectiveness testing (challenge test)}

To determine the effectiveness of the used preservative in the proposed concentration in syrups and drops formulations, the antimicrobial effectiveness testing for Category 3 (oral products, other than antacid, made with aqueous vehicles) was conducted according to USP 35 [10] on two chosen formulations, the syrup (F4) and the drops (F5). These two formulations were chosen based on the taste masking results. In brief, a sufficient volume of test product (typically $10 \mathrm{ml}$ ) was distributed into each of 5 separate containers, and each container was inoculated with a separate test microorganism (Candida albicans, Aspergillus niger, Escherichia coli, Pseudomonas aeruginosa or Staphylococcus aureus). The initial concentration of the viable microorganisms in the test product was determined by standard dilution and plate count methods. Inoculated test products were incubated at $22.5 \pm 2.5$ ${ }^{\circ} \mathrm{C}$ and sampled to determine the microorganism viable count at 7, 14 and 28 day intervals. The microorganism concentration at each interval was compared to the initial concentration, and then preservative effectiveness was determined based USP guidelines. The formula pass the test if the bacterial count is not less than $1.0 \log$ reduction from the initial count at 14 days and no increase from the 14 days and 28 days. For yeast and molds: no increase from the initial calculated count at 14 and 28 days.

\section{Accelerated stability study}

To study the effect of storage on the chosen formulations, an accelerated stability study was conducted, where the formulations (syrups and drops) were packed in well closed glass bottles and stored under stress conditions of $40{ }^{\circ} \mathrm{C}$ and $75 \% \mathrm{RH}$ in a stability cabinet (Binder, Germany) for three months. Samples were withdrawn after 1, 2 and 3 months and examined for some of their physical properties (any changes in $\mathrm{pH}$ and taste as mentioned above).

\section{Statistical analysis}

To compare means of bitterness intensity scores of the samples under study compared to their corresponding control, unpaired t-test was carried out at a 5\% significance level using Microsoft Excel 2007 software.

In-house preliminarily studies were performed to study the effects of cyclodextrin (CD) types and concentrations using natural CDs ( $\alpha-C D, \beta-C D$ and $\gamma-C D$ ) and $\beta$-CD derivatives (Randomly methylated $\beta-C D$, hydroxy propyl $\beta$-CD and sulfobutyl ether $\beta$-CD) with Cetirizine in molar ratio of 1:1, 1:2 and 1:3 in solutions. The concentration of Cetirizine in the test samples were 1 $\mathrm{mg} / \mathrm{ml}$ and these test sample were subjected to human gustatory sensation test to select the most effective CD in the proper molar ratio to inhibit the bitterness scores for Cetirizine. The results revealed that $\alpha$-CD was superior compared with $\beta$-CD and $\gamma$-CD in terms of inhibiting the bitterness scores, whereas randomly methylated (RM) $\beta$ - CD was the most effective among the used $\beta$ - CD

\section{Rerivatieses. . Discussion}

For natural CDs, there were no significant differences $(\mathrm{P}>0.05)$ in the bitterness scores for $\beta$ - and $\gamma$-CDs. On contrary, the bitterness scores for $\alpha-C D$ were significantly $(p<0.05)$ lower than $\beta$ - and $\gamma$-CDs. These results indicate a stronger hydrophobic interaction of $\alpha$-CDs and the drug. As the cavity size increases ( $\beta$ - and $\gamma$-CDs), such interaction is likely to be weaker and the drug molecule may slip easily outside the CD cavity.

For $\beta$ - CD derivatives, RM $\beta$-CD demonstrated to be the most effective in inhibiting the bitterness scores for Cetirizine, whereas hydroxy propyl (HP) $\beta$-CD showed the least taste-masking effects; sulfobutyl ether (SB) $\beta$-CD came in the middle in terms of the taste-masking effect.

$\mathrm{RM} \beta-\mathrm{CD}$, although soluble in water, is a hydrophobic $\beta$-CD derivative. The introduced methyl groups decreased the overall polarity of the molecule and possibly facilitate the inclusion of Cetirizine. In addition, the hydrophobicity imparted by the methyl groups may have caused an increase in the strength of interaction between the drug and the CD [11]. On contrast, the increase in the bitter taste perception for HP $\beta$-CD might be attributed to the steric hindrance of the hydroxy propyl group which may block the way in front of different groups of Cetirizine. SBE $\beta$-CD showed a weak taste masking profile similar to that of $\beta$-CD and HP $\beta$-CD at low concentrations (Figure 2). The relatively low bitterness scores for SBE $\beta$-CD, compared with RM $\beta$-CD are attributed to the increased hydrophilicity of the SBE $\beta-C D$ which decreased the possible interaction with Cetirizine. In addition, the presence of the negative charge on the SBE $\beta$-CD molecule may have hindered the inclusion of the drug due to surface adsorption of the positively charged drug, making the molecule incapable of fitting inside the CD cavity. In general, the increase in the molar concentration of CD was in favour of masking the bitterness of the drug in solutions. Higher concentrations of CDs could guarantee better hostguest interactions and equilibrium between the CDs and Cetirizine. Based on the above findings of the screening study, $\alpha-C D$, $\gamma$-CD and RM $\beta$-CD:Cetirizine in 1:3 molar ratio were selected to prepare syrups and drops formulations. 


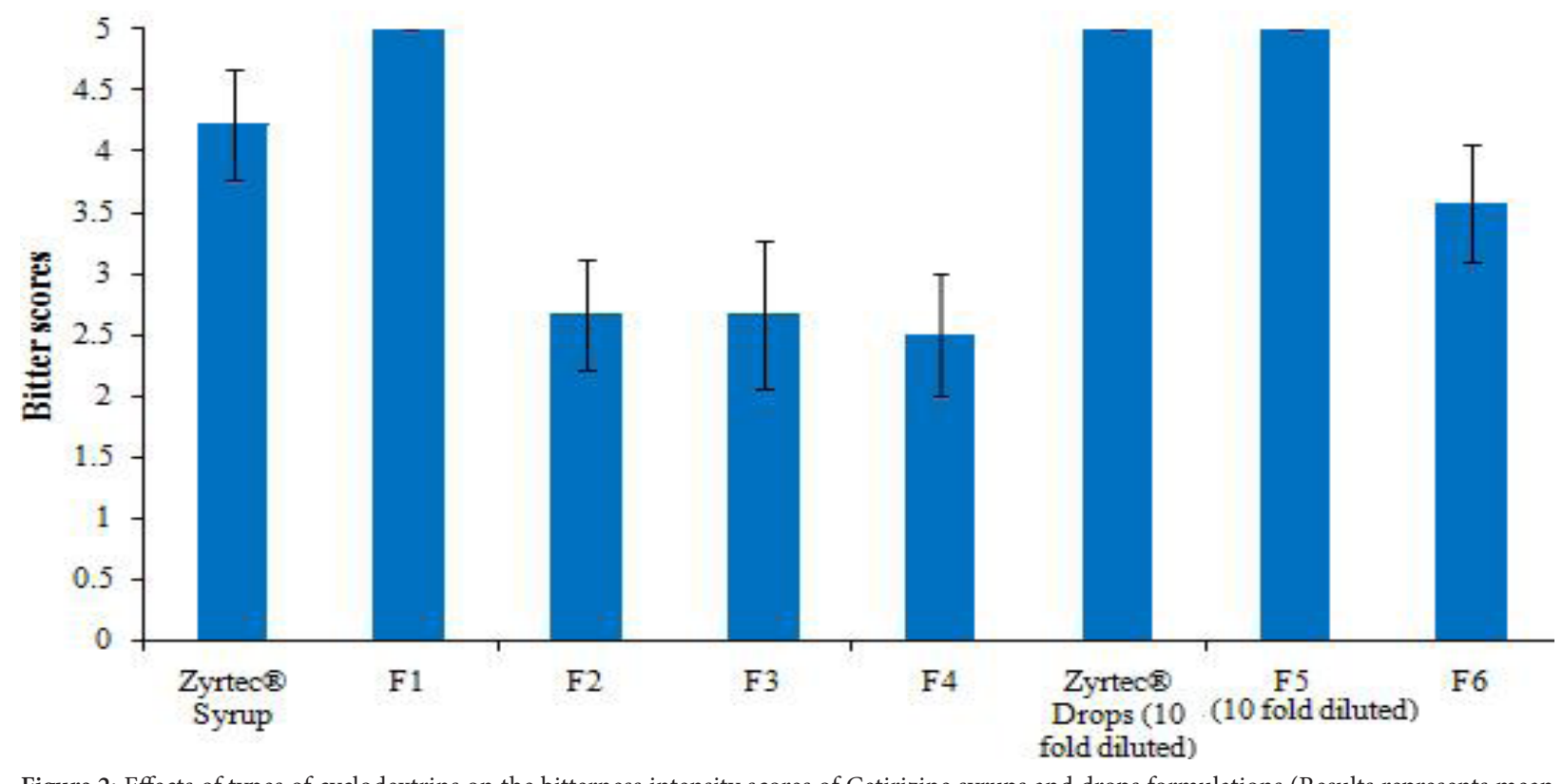

Figure 2: Effects of types of cyclodextrins on the bitterness intensity scores of Cetirizine syrups and drops formulations (Results represents mean values $\pm \mathrm{SD}$ ).

\section{Syrups and drops formulations}

Figure 2 shows the bitterness intensity scores for commercial products (Zyrtec ${ }^{\oplus}$ syrup and drops), control syrups and drops (without CDs), and the prepared syrups and drops formulations containing the selected CDs. The results showed that both commercial products and control formulations scored 4.5 to 5 on human gustatory sensation scores indicating that Cetrizine syrups and drops exhibited extremely bitter taste. Further, both commercial and control drops were too bitter to be scored by the volunteers and it had to be diluted 10 times, nevertheless, it is still extremely bitter. The volunteers unanimously agreed that drops samples were markedly bitterer than the standard quinine solution with bitterness score of 5 . This exceptional bitterness for the drops is due to that the taste sensation depends on concentration, as the drops are 10 times more concentrated than the syrup. Interestingly, RM $\beta$-CD-based drops formulations significantly $(\mathrm{p}<0.01)$ reduced the bitterness intensity score and no further dilution was required, compared with Cetirizine drops without CD and the commercial oral drops.

The syrup formulation prepared with RM $\beta$-CD (F4) continued to be superior over a CD- and $\gamma$ CD-based syrup formulations. These results could be ascribed to the increased lipophilic characteristics imparted by the introduced methyl groups which lead to better interaction and inclusion with the drug [11]. Also, the fact that aggregate formation may entrap the drug molecules and inhibit the interaction with the taste buds. $\gamma$ CD-based syrup formulation showed cloudy precipitation after one week of preparation, it was therefore excluded from further stability studies. The precipitation is most probably due to possible aggregation of $\gamma$-CD in aqueous solutions as reported elsewhere [12]

\section{Determination of $\mathrm{pH}$ and cetirizine content in the taste-masked dosage forms}

Table 2 shows the $\mathrm{pH}$ values of the prepared syrup and drops containing CD and the control preparations. This may be accepted on the basis that the used CDs are neutral and hence will not affect the $\mathrm{pH}$ of the liquid preparation. The calibration graph obtained by plotting the second derivative peak to valley height $(\mathrm{cm})$ against concentration of Cetirizine $(\mathrm{mg} \%)$ showed a linear relationship with a regression coefficient $\left(\mathrm{r}^{2}\right)$ of 0.9999 . It is worthy to note that the second derivative methods were adopted to eliminate the interference between the preservative used, potassium sorbate, and Cetirizine which was observed with the zero order derivative.

\begin{tabular}{|r|r|r|r|}
\hline Formula code & CD used & $\mathrm{pH}$ & Cetirizine content (\%) \\
\hline Syrup & & & \\
\hline F1 & ---- & 4.21 & $95.6 \%$ \\
\hline F2 & $\alpha-C D$ & 4.45 & $97.8 \%$ \\
\hline F3 & $\gamma$-CD & 4.62 & $95.1 \%$ \\
\hline F4 & RM $\beta$-CD & 4.27 & $99.3 \%$ \\
\hline Drops & & & \\
\hline F5 & ---- & 4.10 & $98.1 \%$ \\
\hline F6 & RM $\beta$-CD & 4.23 & $97.5 \%$ \\
\hline
\end{tabular}

Table 2: $\mathrm{pH}$ and content of Cetirizine estimated for the prepared syrup and drops liquid formulations. 
Table 2 shows the results of Cetirizine content (\%) in different Cetirizine dosage forms. As seen, the drug content in the different dosage forms was not affected by the presence of CD in the formulation and all the results were within an accepted range (>95\%). The $\mathrm{pH}$ values recorded for syrups and drops formulations ranged between 4.10 and 4.62 .

Potassium sorbate is the ionizable soluble form of sorbic acid which has a $\mathrm{pKa}$ of 4.8 . At the $\mathrm{pH}$ of the prepared syrups and drops $(\mathrm{pH}=4-5)$, it is mostly present in the active form [13].

\section{Antimicrobial effectiveness testing (challenge test)}

One of the most difficult challenges facing the development of liquid dosage forms using CDs, as a potential excipient to solve biopharmaceutical problems, is the interaction between the $\mathrm{CD}$ and the preservative. CDs have been reported to decrease the antimicrobial activity of many preservatives [14]. This interaction is due to competitive displacement of the drug and preservative molecules on the CD cavity leading to a decrease in the concentration of the free preservative molecules available for the expected antimicrobial activity. Further, as the preservative displaces the drug from the CD cavity, the free drug concentration might exceed the saturated solubility and in some instances lead to precipitation out of the solution. Therefore, the proper choice of the preservative and its concentration could have a direct impact on the success of liquid dosage forms containing CDs.

In the present study, the preservative employed was potassium sorbate. It was preferably chosen over the usual parabens which are reported to interact with CDs and competitively displace the included drug in the CD cavity, accompanied by reduced antimicrobial activity [14].

Selected syrup (F4) and drops (F6) formulations containing RM $\beta$-CD (which showed the best taste-masking results compared to other formulations) were subjected to the microbiological challenge test according to the USP30. Both formulations passed the test indicating that the preservative used in the proposed formulation and concentration was sufficient to inhibit microbiological (bacterial, fungal and pathogens) contamination, if present.

\section{Stability study}

\section{Determination of cetirizine content in stored formulations}

A literature survey was done to find a stability indicating method for Cetirizine in presence of CDs but none was found. However, stability indicating method based on HPLC for Cetirizine in conventional marketed formulations was published by Paw et al. [15]. In the HPLC method, extraction solvent was methanol, the samples were chromatographed on a RP-C18 column and UV detected at $227 \mathrm{~nm}$. The elution was achieved isocratically with a mobile phase of $0.067 \mathrm{M}$ phosphate buffer $\mathrm{pH} 3.40 / \mathrm{acetonitrile}(1: 1 \mathrm{v} / \mathrm{v})$.

Employing this method on control formulations showed (Figure 3) a retention time of 4 minutes and a high recovery of Cetirizine $(101 \% \pm 0.99$ for syrup and $100.4 \% \pm$ for drops), however, all CD containing formulations gave fluctuating results that were not trustful. Minor changes to readjust the method by changing the $\mathrm{pH}$ of the mobile phase or changing the extraction solvent did not give any improvement. As CD themselves may interfere with the assay method, further studies required to determine the content of Cetirizine in CD containing formulations are beyond the scope of this study.

The tested control formulations showed high recovery of Cetirizine over three months under stress storage conditions $\left(40{ }^{\circ} \mathrm{C}\right.$ and $75 \% \mathrm{RH}$ ) indicating good chemical stability of the drug. CDs are not expected to produce stability problems in the prepared formulations as CDs have been used as a stabilizer against common degradation and oxidation due to drug inclusion and shielding inside hydrophobic cavities of the host CD. Cetirizine is not expected to pose stability problems in the prepared formulations.

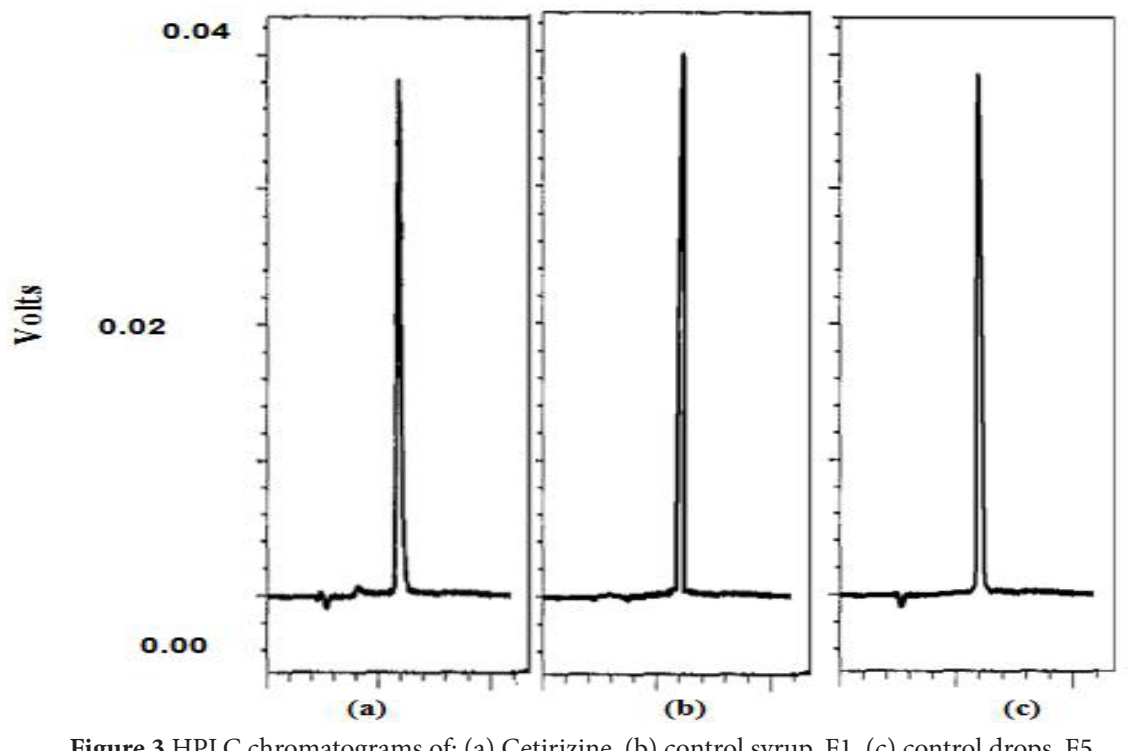

Figure 3 HPLC chromatograms of: (a) Cetirizine, (b) control syrup, F1, (c) control drops, F5. 
Bitter taste intensity of dosage forms under study

Table 3 and Figure 4 show the results of the changes of $\mathrm{pH}$ and bitter intensity scores for the syrups and drops formulations stored under stress conditions of $40{ }^{\circ} \mathrm{C}$ and $75 \%$ R.H. for a period of 3 months.

\begin{tabular}{|c|c|c|c|c|c|}
\hline \multirow{2}{*}{ Formula code } & \multirow{2}{*}{ CD used } & \multicolumn{4}{|c|}{$\mathrm{pH}$} \\
\cline { 3 - 6 } & & Zero time & One month & Two months & Three months \\
\hline Syrup & & & & & 4.28 \\
\hline F1 & ---- & 4.21 & 4.32 & 4.48 & 4.56 \\
\hline F2 & $\alpha-C D$ & 4.45 & 4.39 & 4.36 & 4.35 \\
\hline F4 & RM $\beta-C D$ & 4.27 & 4.41 & & 4.25 \\
\hline Drops & & & & 4.24 & 4.29 \\
\hline F5 & ---- & 4.10 & 4.22 & 4.22 & \\
\hline F6 & RM $\beta-C D$ & 4.23 & 4.31 & & \\
\hline
\end{tabular}

Table 3: $\mathrm{pH}$ of different Cetirizine liquid dosage forms stored under stress conditions $\left(40^{\circ} \mathrm{C}\right.$ and $\left.75 \% \mathrm{RH}\right)$ for three months.

The results showed that a CD-based syrup formulation (F2) showed relatively stable taste profiles over three months unlike a fluctuating profiles for RM $\beta$-CD-based syrup (F4) whose bitter taste slightly reduced by time (Figure 4). The peculiar taste profile of RM $\beta$-CD may be explained by the effect of the stress storage conditions. The high temperature environment may have allowed better inclusion of Cetirizine into the hydrophobic cavity of the RM $\beta$-CD. By increasing the time of storage under such conditions, the amount of dissolved RM $\beta$-CD may have increased. Consequently, the inclusion complexation and amount of formed aggregates may have increased accounting for the improvement in taste by time especially in Syrupy environments (a highly concentrated vehicle of polyols). Unlike the case in syrups, RM $\beta$-CD showed stable taste profiles in the Drops preparations (Figure 4). This could support the hypothesis of solubility factor of RM $\beta-C D$ in various vehicles.

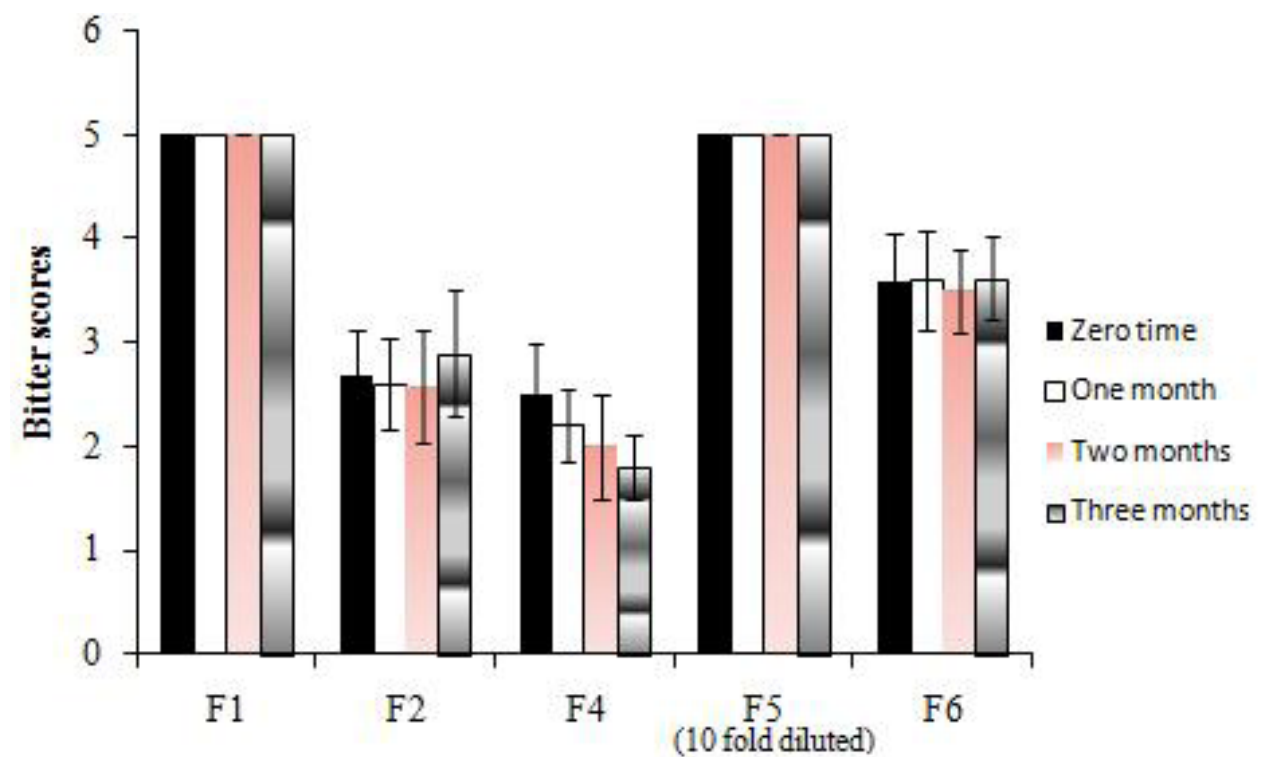

Figure 4: Average bitter intensity scores of the prepared syrups and drops stored under stress conditions $\left(40{ }^{\circ} \mathrm{C}\right.$ and $75 \% \mathrm{RH})$ for one, two and three months.

\section{Measurements of $\mathrm{pH}$ of syrups and drops formulations}

Table 3 shows the results of $\mathrm{pH}$ of the prepared formulations after storage under stress conditions for 3 months. The results showed that stress storage conditions did not affect the $\mathrm{pH}$ of the prepared samples. The CDs used were neutral and showed $\mathrm{pH}$ results similar to those of blank preparations indicating that the presence of neutral CDs in liquid preparations may probably have negligible effect on their $\mathrm{pH}$ and favourably maintain the preservative in the active form over the storage period. These results support those recorded for the antimicrobial activity test.

\section{Conclusion}

Natural cyclodextrins (CDs) and $\beta$ CD derivatives were utilized as potential excipients to solve a palatability issue for pediatric oral liquids of Cetirizine. Both Cetirizine syrup and drops require taste masking of bitterness of the drug in order to enhance patient acceptability. RM $\beta$ CD dramatically reduced the bitterness intensity scores of Cetirizine using the human gustatory sensation test. RM $\beta$ CD-based syrup and drops formulations were significantly palatable and stable in terms of keeping their palatability as well as preservation properties under stress storage conditions suggesting their potential as agreeable oral liquids for pediatric patients. 


\section{References}

1. Sweetman SC (2007) Martindale: The Complete Drug Reference, 35th edn. Pharmaceutical Press and Royal Pharmaceutical Society of Great Britain, London.

2. Center for Drug Evaluation and Research (2007) Pediatric Exclusivity Labeling Changes-Updates, USA.

3. Ono N, Miyamoto Y, Ishiguro T, Motoyama K, Hirayama F, et al. (2011) Reduction of bitterness of antihistaminic drugs by complexation with $\beta$-cyclodextrins. J Pharm Sci 100: 1935-43.

4. El Massik MA (2006) Development of cetirizine chewable tablets with inhibited bitterness using liquisolid compact technology. Alex J Pharm Sci 20: 11-6.

5. Sohi H, Sultana Y, Khar RK (2004) Taste masking technologies in oral pharmaceuticals: recent developments and approaches. Drug Dev Ind Pharm 30: 429-48.

6. Yoshimi S, Kiyomi S, Yasuhiko N, Hiroshi F, Hirokazu M (1991) Rapid-releasing oral particle pharmaceutical preparation with unpleasant taste masked. European Patent 1991.

7. Gerard C, Etienne B, Edouard G (1993) Rapidly disintegratable multiparticulate tablet. European Patent 1993.

8. Ishizaka T, Okada S, Tokuyama E, Mukai J, Uchida T (2008) Suppression of bitterness and improvement of palatability of commercial prednisolone powder. Chem Pharm Bull 56: 1395-9.

9. El-Gindy A, Emara S, Hadad GM (2004) Determination of certain drugs in binary mixtures formulations by second derivative ratio spectrophotometry and LC. Farmaco 59: 703-12.

10. USP 35/ NF 30 (2012) United States Pharmacopeia 35. The United States Pharmacopeial Convention, Rockville, USA.

11. Loftsson T, Magnusdottir A, Masson M, Sigurjonsdottir JF (2002) Self-association and cyclodextrin solubilization of drugs. J Pharm Sci 91: 2307-16.

12. Irie T, Uekama K (1997) Pharmaceutical applications of cyclodextrins. III. Toxicological issues and safety evaluation. J Pharm Sci 86: 147-62.

13. Bell TA, Etchelis JL, Borg AF (1959) Influence of sorbic acid on the growth of certain species of bacteria, yeasts, and filamentous fungi. J Bacteriol 77: 573-80. 14. Loftsson T, Stefansdouir O, Frioriksdouir H, Guomundsson O (1992) Interactions between preservatives and 2-hydroxypropyl $\beta$-cyclodextrin. Drug Dev Ind Pharm 18: 1477-84.

15. Paw B, Misztal G, Hopkala H, Drozd J (2002) Development and validation of a HPLC method for the determination of cetirizine in pharmaceutical dosage forms. Pharmazie 57: 313-5. 